\title{
Actitud ante el COVID-19 y su vacuna, de estudiantes de ESO y Bachillerato en España
}

Jorge Pozuelo Muñoz - Universidad de Zaragoza

0000-0002-9223-6832

Recepción: 23.11.2021 | Aceptado: 30.12.2021

Correspondencia a través de ORCID: Jorge Pozuelo Muñoz

iD 0000-0002-9223-6832

Citar: Pozuelo Muñoz, J (2022). Actitud ante el COVID-19 y su vacuna, de estudiantes de ESO y Bachillerato en España. REIDOCREA, 11(1), 1-13.

Resumen: En la situación de pandemia actual, conocer la percepción del riesgo ante el virus, y la intención de conducta ante la vacuna contra el mismo, puede aportar conocimiento valioso para tomar decisiones tanto en el contexto educativo escolar, como en otros ámbitos. Para avanzar en el conocimiento sobre el tema, se ha desarrollado un estudio cuantitativo donde se han analizado las respuestas de 308 estudiantes de ESO y Bachillerato de toda España, ante un cuestionario validado previamente. Esto refuerza el conocimiento epistemológico de la percepción del alumnado ante cuestiones sociocientíficas de actualidad y presentes en el debate público en todas sus escalas, también dentro del aula. A su vez, se busca abrir la puerta a futuros estudios, que, en comparativa con los resultados de este trabajo y otros similares, permitan establecer qué factores afectan a las actitudes del alumnado hacia la ciencia y en qué medida, sobre todo, aquellos que permitan arrojar luz sobre la influencia de la enseñanza-aprendizaje en ciencias en el aula frente al aprendizaje por otros medios no contrastados. Los resultados muestran distribución de la percepción del riesgo y de la intención de vacunación en función de la edad y del género.

Palabra clave: COVID-19

Attitude towards COVID-19 and its vaccine, of ESO and Baccalaureate students in Spain

\begin{abstract}
We are in a pandemic situation in which knowing the perception of risk of the virus, and the intention of conduct before the vaccine against it, can provide valuable knowledge to make decisions both in the educational context of the school and in other areas. To advance knowledge on the subject, a quantitative study has been developed in which the responses of $308 \mathrm{ESO}$ and Baccalaureate students, from all over Spain, have been analysed, by means of a previously validated questionnaire. This reinforces the epistemological knowledge of the students' perception of current socio-scientific issues that are present in the public debate at all its scales, also within the classroom. In turn, it seeks to open the door to future studies, which, in comparison with the results of this work and other similar ones, allow to establish what factors affect students' attitudes towards science and to what extent, especially those who allow shedding light on the influence of teaching-learning in science in the classroom compared to learning by other untested means. The results show a distribution of the risk perception and the vaccination intention according to age and gender.
\end{abstract}

Keyword: COVID-19

\section{Introducción}

Durante mucho tiempo, desde el campo de investigación de la didáctica de las ciencias, hemos estado preocupados por analizar las actitudes hacia la ciencia de estudiantes de educación primaria (EP), educación secundaria y bachillerato (en adelante, ESO-Bto). El objetivo final de algunos de estos estudios ha sido comprender las repercusiones de las materias de ciencias, ya sea en las decisiones del alumnado sobre su futuro formativo (Osborne, Simon y Collins, 2003) o en la percepción de los alcances de la ciencia y la tecnología en su globalidad (Acevedo et al., 2005). Actualmente, con la pandemia de COVID-19 que estamos viviendo, la actitud hacia la ciencia del alumnado de estas edades puede verse modificada si atendemos a situaciones concretas que les afectan y que están apoyadas por la ciencia, como es en este caso, la vacunación contra el coronavirus. 
Dado que la vacunación de la población de estas edades es inminente, previo al comienzo de este plan de vacunación, nos interesa conocer la opinión de los estudiantes de ESO-Bto, con edades comprendidas entre los 12 y los 18 años, sobre el coronavirus y su vacuna. Para ello, se ha diseñado un cuestionario ad hoc de preguntas tipo test.

El objetivo de este trabajo, por lo tanto, ha sido conocer la percepción que estos alumnos tienen sobre el coronavirus, su actitud hacia las vacunas y en concreto hacia las del COVID-19, y su intención de conducta hacia la vacunación.

Uno de los objetivos históricos de la investigación en didáctica de las ciencias ha sido conocer las bases de la toma de decisiones, y/o la intención de conducta de los alumnos y su relación con la ciencia escolar (Osborne, Simon y Collins, 2003). Durante mucho tiempo, se investigó para intentar entender cómo esta toma de decisiones concluía en que los alumnos no elegían carreras científicas (Osborne, Simon y Collins, 2003) dando lugar a un conocimiento valioso sobre la enseñanza de la ciencia en la escuela. Algunas de las conclusiones encontradas ponían el foco en la educación propedéutica de la ciencia en la escuela, es decir, que los alumnos recibían una educación focalizada a la consecución de resultados y poco a una enseñanza de la ciencia útil y aplicable en contextos reales (Vázquez-Alonso, Acevedo-Díaz y Manassero-Mas, 2005). También Duit y Pfund (1998) concluyeron en su estudio que los alumnos aplican poco los conocimientos que han adquirido en la escuela en situaciones reales debido al mismo motivo y, como consecuencia, es sabido que si los estudiantes no perciben la utilidad de la ciencia en su vida cotidiana pierden el interés por la misma (Reiss, 2004; George, 2006). Lupión-Cobos y Girón-Gambero (2020) encontraron en su estudio que, a pesar de que los alumnos de estas edades no muestran excesivo interés por la ciencia en su tiempo de ocio, sí muestran una concienciación ciudadana importante sobre los temas científicos. Y Vázquez y Manassero (2008) decían que, conforme aumenta la edad de los estudiantes, se distingue una tendencia según el género. Las mujeres se desinteresan más rápidamente por las materias científicas que los hombres, sin embargo, destaca que, cuando la ciencia trabajada tiene una aplicación directa en la sociedad, las mujeres tienden a retomar el interés por la ciencia.

Una forma de trabajar las ciencias en el aula que puede despertar el interés del alumnado, por percibir la utilidad de la ciencia, es a través del planteamiento de controversias sociocientíficas. De acuerdo con Hodson (2020) la enseñanza de las ciencias debe abordar cuestiones sociocientíficas con los estudiantes y prepararlos para ser ciudadanos informados científicamente y poder tomar decisiones fundamentadas. Construir conocimiento científico en el aula a través de controversias sociocientíficas favorece la actitud hacia la ciencia de los alumnos, además del pensamiento crítico, la capacidad de argumentación y de toma de decisiones (Sadler, 2011; Díaz y Jiménez-Liso, 2012). Al mismo tiempo las controversias científicas son intrínsecas a la construcción de conocimiento científico y muestran el conflicto científico como un proceso común en la ciencia (Acebedo-Díaz y García-Carmona, 2017).

Actualmente, nos encontramos en un contexto de pandemia mundial, donde la formación científica tiene una importancia clave ante la actitud y la intención de 
conducta frente al virus y frente a la vacuna desarrollada contra el mismo (Varela, López-Gómez, Fraguela-Vale, 2021). Esta situación de pandemia ha acercado la ciencia a la vida cotidiana y en este aspecto, los alumnos han percibido la utilidad de la ciencia en el sentido más inmediato. En poco más de un año, gracias a la ciencia se han conseguido desarrollar vacunas específicas contra el virus que nos afecta. $Y$, en este momento, muchos países nos encontramos en el punto de vacunar a la población para protegernos ante el SARS-CoV-2. Esto pone de manifiesto que las vacunas y la vacunación suponen una cuestión sociocientífica, ya que la decisión que se tome frente a ellas va más allá de las consideraciones científicas, al ponerse en juego valores, creencias personales y la influencia social (Uskola, Burgoa y Maguiregi, 2021). Estas controversias involucran aspectos científicos, pero también políticos, éticos, ambientales o económicos (Jiménez-Aleixandre, 2010), y tratarlos en clase puede movilizar gran variedad de conocimientos, valores y capacidades que son, además, contextualizados (Sadles y Zeidler, 2005; Sadler, 2011).

Lupión-Cobos y Girón-Gambero (2020) encontraron indicios de que los estudiantes no logran integrar claramente la reflexión y la autocrítica ante la toma de decisiones. Dichos autores señalan que, debido a que este aspecto no está muy incorporado en los programas educativos, no llega a trascender a los estudiantes. De acuerdo a Girón, Lupión y Blanco (2020), este hecho podría abordarse en mayor profundidad en la formación de los estudiantes, utilizando propuestas innovadoras ya que, en la sociedad actual, es necesario que se promueva una movilización de capacidades y competencias científicas. Blanco, España y Rodríguez (2012) y Solbes et al. (2012), proponen que la innovación en su formación se realice a través de innovaciones que permitan la contextualización de los contenidos o el tratamiento STEM. Acebedo-Díaz y García Carmona (2017) afirman que en el uso de las controversias sociocientíficas la reducción de la brecha entre el conocimiento producido (en nuestro caso los efectos de la vacuna) y la ciencia en fase de descubrimiento (en nuestro caso la fabricación de la vacuna) ofrece un marco idóneo sobre la complejidad en la construcción de conocimiento científico. Por ello, debido a la premura con la que la circunstancia sanitaria cambia y con ello las actitudes hacia la vacuna de la sociedad y en nuestro caso el alumnado, se ha decidido realizar un estudio para dejar constancia de las actitudes del alumnado frente a la vacuna cuando aún no se conocen los efectos de dicha vacunación.

De acuerdo a Díaz y Jiménez-Liso (2012) entre las temáticas sociocientíficas, los temas relacionados con la salud no son los temas más trabajados en las aulas a pesar de ser los que más frecuentemente aparecen en los medios de comunicación, y de los que más interés despiertan entre los estudiantes, por lo que deberían trabajarse más en el contexto escolar. No hay que olvidar que lo que se trabaja en el aula y la manera de tratarlo influye, entre otros, sobre la actitud de los alumnos ante la ciencia (Acebedo-Díaz y García Carmona, 2017).

En las circunstancias de pandemia en que nos encontramos, el alumnado formado en ciencia escolar tiene la oportunidad de aplicar esos conocimientos a una situación real. Es decir, pueden basar su toma de decisiones (y/o su intención de conducta en el caso de menores de edad) ante la enfermedad COVID-19 y su vacuna en su formación escolar previa. 
Algunos autores están empezando a investigar sobre la actitud de los estudiantes ante la vacuna. Por ejemplo, Rodríguez-Losada et al. (2021) presentan una primera aproximación sobre el estudio de factores que pueden influir en la toma de decisiones sobre la vacunación frente a la COVID-19 de futuros maestros de educación infantil. En su estudio, estos autores concluyen que el alumnado de la muestra presenta un conocimiento ingenuo sobre diversas dimensiones relativas a la vacuna y la vacunación, cuya adecuada comprensión es necesaria para la toma de decisiones responsables. Además, indican que el auge de las noticias falsas en los medios de comunicación durante el tiempo de pandemia (Puig, Blanco-Anaya y Pérez-Maceira, 2021) condiciona y/o ha condicionado la toma de decisiones responsables sobre la vacunación ante el virus (Rodríguez-Losada et al., 2021). En los resultados de su investigación, Rodríguez-Losada et al. (2021) concluyen en la desconfianza hacia la ciencia, en particular hacia la vacuna del COVID-19 de los alumnos de su muestra de estudio. Esto seguramente sea debido a la percepción de "ciencia express" que tienen los alumnos en relación al desarrollo y validación de la vacuna (LópezGoñi, 2020).

El concepto de actitud hacia la ciencia es algo confuso, a menudo mal articulado y no bien entendido (Osborne et al., 2003). En 1975, Gardner (Gardner, 1975) aportó algo de claridad, diferenciando las actitudes hacia la Ciencia y las actitudes científicas y, fue Klopfer (1971) quien estableció la definición de actitudes hacia la Ciencia como "los sentimientos, creencias y valores que se tienen sobre un objeto que puede ser la empresa de la ciencia, la ciencia escolar, el impacto de la ciencia en la sociedad o los propios científicos". Según Gardner (1975) las actitudes hacia la ciencia son las respuestas emocionales hacia lo científico. En relación a la influencia del género, Lupión-Cobos y Girón-Gambero (2020) encontraron que las chicas parecen adoptar actitudes científicas más favorables que los chicos, a pesar de que esto no se corresponda con la posterior formación científica superior que escogen, en la que las chicas eligen en una menor proporción cursar carreras de ciencias que los chicos (Vázquez y Manassero, 2009).

Una de las propuestas de futuro de Lupión-Cobos y Girón-Gambero (2020) ha sido realizar estudios de diagnóstico sobre las percepciones de los estudiantes hacia la ciencia, de manera que los resultados sirvan para proyectar objetivos concretos de educación científica en la escuela orientados a formar ciudadanos alfabetizados en ciencia. Con este trabajo, queremos aportar nuestros resultados a ese propósito. Por ello, el objetivo principal de este trabajo es conocer más sobre la actitud ante el virus COVID-19, ante la vacuna frente a éste y ante las decisiones (toma de decisiones y/o intención de conducta) en relación a la vacunación, de los alumnos de ESO y Bto formados en ciencia escolar. Y, en segundo lugar, estudiamos la influencia del género y del curso escolar de los estudiantes sobre esta actitud e intenciones de conducta frente al virus y su vacuna.

\section{Método}

En este estudio hemos utilizado un cuestionario diseñado ad hoc para conocer más sobre la actitud de los alumnos en torno al COVID-19 y su vacuna. 
Seguimos por tanto la metodología de encuestas, ampliamente utilizada en el ámbito de estudio de la didáctica de las ciencias experimentales (de Pro y Pérez, 2014; Mazas y Bravo, 2018). Esta metodología permite a los investigadores recoger datos y hacerlo sin intervenir, ni introducir cambios en los datos que se van a recoger (actitudes y percepciones) (Morales, 2000; Fontes de Gracia et al., 2008). La toma de datos se ha llevado a cabo en las semanas previas al comienzo de la vacunación en España (noviembre y diciembre de 2020) para evitar el sesgo de conocimiento que podría producir conocer los efectos de la vacunación.

Muestra. El cuestionario se pasó en formato Google Forms, a un total de 308 alumnos de cursos entre $1^{\circ}$ de ESO y $2^{\circ}$ de Bachillerato, de distintas Comunidades Autónomas de toda España. En la muestra participaron un total de 133 mujeres, 162 hombres y 13 personas identificadas con otros géneros.

Tabla 1. Distribución de los participantes según el curso y el género

\begin{tabular}{lcccccc}
\hline Género & \multicolumn{7}{c}{ Curso } \\
\hline & $1^{\circ}$ ESO & $2^{\circ}$ ESO & $3^{\circ}$ ESO & $4^{\circ}$ ESO & $1^{\circ}$ Bto & $2^{\circ}$ Bto \\
\hline Femenino & 36 & 14 & 24 & 25 & 15 & 19 \\
Masculino & 45 & 34 & 29 & 14 & 13 & 27 \\
Otros & 3 & 8 & 0 & 0 & 0 & 2 \\
\hline
\end{tabular}

\section{Instrumento}

Antes de diseñar los ítems se tuvo en cuenta el estudio de Toma (2020) en el que analiza una serie de instrumentos utilizados en el estudio de las actitudes hacia la ciencia de los alumnos. En su trabajo concluye que, para que podamos dar por válidos los resultados, la muestra debe ser de $n>100$, el diseño metodológico del instrumento debe ser riguroso, debe ser validado y deben contener preguntas de componentes afectivos, cognitivos y conductuales, entre otras cosas.

Se tuvieron en cuenta los puntos descritos por Toma (2020) por lo que, en primer lugar, se diseñó un cuestionario inicial que fue validado por tres expertos en el área de conocimiento de la Biología, y a continuación, se plantearon los 10 ítems que se muestran en la tabla siguiente, agrupados en tres categorías. En la primera categoría (color naranja) se analiza la percepción que los alumnos tienen de las vacunas en general y de la vacuna frente al COVID-19 en particular y se recoge en las preguntas 1, 2, 3 y 4 . Una segunda categoría (color azul) incluye las preguntas 5 y 6 , que abordan la percepción ante el riesgo de contraer la enfermedad relacionado con el virus COVID-19. Por último, la tercera categoría engloba las preguntas de la 7 a la 10 (color verde), que valoran la actitud ante la vacuna del COVID-19.

En la Tabla 2 se pueden consultar las cinco opciones de respuesta codificadas como A, B, C, D y E. Las respuestas se han codificado de esta manera con el único fin de que el análisis de las respuestas resulte más sencillo. De esta manera, por ejemplo, en las dos primeras preguntas las respuestas son equivalentes, de manera que la $A$, se corresponde con la misma respuesta tanto 
en la pregunta 1 como en la 2 y así con las cinco opciones de respuesta. Esto nos permite comparar las respuestas obtenidas en ambas preguntas.

Tabla 2. Cuestionario de preguntas que completaron los estudiantes.

\begin{tabular}{|c|c|c|c|}
\hline $\mathrm{N}^{0}$ & Cuestión & Respuestas & Código \\
\hline \multirow[t]{5}{*}{1} & \multirow[t]{5}{*}{$\begin{array}{l}\text { ¿Para qué sirven las } \\
\text { vacunas? }\end{array}$} & $\begin{array}{l}\text { Para evitar el contagio de un virus en tu cuerpo y si te contagias, } \\
\text { que los síntomas sean leves }\end{array}$ & A \\
\hline & & Para sanar los síntomas de un virus ya contraído & B \\
\hline & & $\begin{array}{l}\text { Para evitar que puedas contagiar a alguien de un virus que has } \\
\text { contraído y evitar los síntomas }\end{array}$ & C \\
\hline & & $\begin{array}{l}\text { Para evitar tu contagio, sanar los síntomas que ya tienes y } \\
\text { evitar contagio a los demás }\end{array}$ & $\mathrm{D}$ \\
\hline & & No lo sé & $\mathrm{E}$ \\
\hline \multirow[t]{5}{*}{2} & \multirow{5}{*}{$\begin{array}{l}\text { ¿Para qué sirve la } \\
\text { vacuna } \\
\text { frente al Covid-19? }\end{array}$} & $\begin{array}{l}\text { Para evitar que te contagies del Covid y si te contagias, que los } \\
\text { síntomas sean leves }\end{array}$ & A \\
\hline & & Para sanar los síntomas del Covid una vez te has contagiado & B \\
\hline & & $\begin{array}{l}\text { Para evitar que contagies a alguien cuando te has contagiado del } \\
\text { Covid }\end{array}$ & C \\
\hline & & $\begin{array}{l}\text { Para evitar que te contagies y si te contagias, sanar los } \\
\text { síntomas y evitar el contagio a los demás }\end{array}$ & D \\
\hline & & No lo sé & $E$ \\
\hline \multirow[t]{4}{*}{3} & \multirow{4}{*}{$\begin{array}{l}\text { ¿Crees que las vacunas } \\
\text { son útiles a } \\
\text { día de hoy? }\end{array}$} & Sí, siguen evitando la propagación de muchas enfermedades & A \\
\hline & & No, creo que son un invento de los gobiernos y las farmacéuticas & B \\
\hline & & No, hoy en día hay muy pocas enfermedades infecciosas & C \\
\hline & & $\begin{array}{l}\text { No, si me contagiara de alguna enfermedad, entonces iría al } \\
\text { médico para curarme }\end{array}$ & $\mathrm{D}$ \\
\hline \multirow[t]{5}{*}{4} & \multirow{5}{*}{$\begin{array}{l}\text { ¿Cuál es tu opinión sobre } \\
\text { la vacuna } \\
\text { contra el Covid? }\end{array}$} & $\begin{array}{l}\text { Creo que la vacuna es segura porque ha pasado muchos } \\
\text { controles sanitarios }\end{array}$ & A \\
\hline & & $\begin{array}{l}\text { Creo que no se conocen los efectos adversos de la vacuna y } \\
\text { puede ser peligrosa }\end{array}$ & B \\
\hline & & Dudo que la vacuna sea eficaz y además puede ser peligrosa & C \\
\hline & & Creo que la vacuna es una mentira de los gobiernos & D \\
\hline & & No tengo opinión sobre la vacuna & $E$ \\
\hline \multirow[t]{5}{*}{5} & \multirow{5}{*}{$\begin{array}{l}\text { ¿Crees que tienes riesgo } \\
\text { de } \\
\text { contagiarte de Covid-19? }\end{array}$} & Sí, creo que tengo riesgo de contagiarme & A \\
\hline & & No, porque soy joven y los jóvenes casi no se contagian & B \\
\hline & & No, porque cumplo con las normas de seguridad & C \\
\hline & & No, porque soy joven y casi nunca me pongo enfermo & D \\
\hline & & No, porque ya he tenido Covid & E \\
\hline \multirow[t]{5}{*}{6} & \multirow{5}{*}{$\begin{array}{l}\text { Indica qué situación } \\
\text { crees qué es de riesgo } \\
\text { para contagiarse del } \\
\text { Covid-19 }\end{array}$} & $\begin{array}{l}\text { Compartir tu clase con las ventanas cerradas, pero usando } \\
\text { mascarilla }\end{array}$ & A \\
\hline & & $\begin{array}{l}\text { Compartir tu clase con todas las ventanas abiertas y usando } \\
\text { mascarillas }\end{array}$ & B \\
\hline & & Estar al aire libre sin usar mascarillas & C \\
\hline & & Todas son de riesgo & $\mathrm{D}$ \\
\hline & & Ninguna es de riesgo & $E$ \\
\hline \multirow[t]{6}{*}{7} & \multirow[t]{6}{*}{$\begin{array}{l}\text { ¿Te pondrías la vacuna } \\
\text { contra el Covid? }\end{array}$} & $\begin{array}{l}\text { Sí, es necesario vacunarme porque me protege totalmente } \\
\text { contra la enfermedad producida por el Covid }\end{array}$ & A \\
\hline & & Necesito tener más información para saber si debo vacunarme & $B$ \\
\hline & & No, creo que los efectos adversos pueden ser peligrosos & C \\
\hline & & No, sería caer en la trampa de los gobiernos & $\mathrm{D}$ \\
\hline & & $\begin{array}{l}\text { Sí, pero esperaría un tiempo para ver los efectos en otras } \\
\text { personas }\end{array}$ & $E$ \\
\hline & & Sí & A \\
\hline
\end{tabular}


Si tu respuesta a la pregunta 8 ha sido que no: ¿Por qué?

Sí tu respuesta a la pregunta 8 ha sido sí: ¿Por qué?
No frente al Covid?

No tengo dudas sobre la seguridad de la vacuna.

Aunque tenga algo de miedo a los efectos adversos, creo que evitará peligros mayores

Me vacuno por si acaso, aunque creo que la vacuna no es eficaz

Aunque no creo que sirva para mí, ayudaré a que el virus no se siga propagando

No creo que me contagie de Covid

Estoy sano y si me contagio no creo que sea tan grave

La vacuna no es eficaz y no se han hecho suficientes pruebas

La vacuna es una estafa de los gobiernos y las farmacéuticas
B

A

B

C D A B C

D

\section{Resultados}

Las respuestas al cuestionario de los estudiantes se han clasificado según el curso y según el género de los alumnos encuestados. El género se ha clasificado como $\mathrm{M}$ (mujer), $\mathrm{H}$ (hombre y $\mathrm{O}$ (otros). Las respuestas a las preguntas se analizan según las categorías establecidas comentadas anteriormente.

\section{Categoría 1: Percepción general frente a las vacunas y percepción frente a la vacuna específica contra el COVID-19}

En la Tabla 3 se muestra la distribución porcentual de las respuestas a la pregunta 1: ¿para qué sirven las vacunas?

Tabla 3. Porcentajes de respuestas a la pregunta 1, en función del curso.

\begin{tabular}{lcccccc}
\hline & $1^{\circ} \mathrm{ESO}$ & $2^{\mathrm{a} E S O}$ & $3^{\circ} \mathrm{ESO}$ & $4^{\circ} \mathrm{ESO}$ & $1^{\circ} \mathrm{Bto}$ & $2^{\circ} \mathrm{Bto}$ \\
\cline { 2 - 7 } $\mathrm{A}$ & $46.4 \%$ & $46.4 \%$ & $52.8 \%$ & $43.7 \%$ & $57.1 \%$ & $45.8 \%$ \\
$\mathrm{~B}$ & $2.4 \%$ & $8.9 \%$ & $0 \%$ & $5.1 \%$ & $3.6 \%$ & $2.1 \%$ \\
$\mathrm{C}$ & $7.1 \%$ & $7.1 \%$ & $5.7 \%$ & $5.1 \%$ & $0 \%$ & $14.6 \%$ \\
$\mathrm{D}$ & $42.9 \%$ & $35.7 \%$ & $41.5 \%$ & $41.0 \%$ & $39.3 \%$ & $33.3 \%$ \\
$\mathrm{E}$ & $1.2 \%$ & $1.9 \%$ & $0 \%$ & $5.1 \%$ & $0 \%$ & $4.2 \%$ \\
\hline
\end{tabular}

Analizando las respuestas por cursos, en todos, casi la mitad de los estudiantes seleccionan esta opción A según la cual creen que las vacunas sirven para evitar el contagio de un virus y atenuar los síntomas en caso de contagio. En el caso de 1 을 Bto el porcentaje supera el $57 \%$. Otra gran parte de los alumnos (39.3\%) escoge la opción $D$, es decir, piensan que las vacunas sirven para evitar el contagio del virus, sanar los síntomas y evitar contagiar a los demás. Esta respuesta la escogen un total de alumnos que va en porcentaje desde un $3.7 \%$ del total de $2^{\circ}$ de ESO hasta un $42.9 \%$ de los alumnos de $1^{\circ}$ de ESO.

En cuanto al análisis por género, eligen la respuesta A 148 alumnos de los 308 encuestados, es decir, un $48 \%$ del total, de los cuales, un $43.9 \%$ son mujeres, un $52.7 \%$ son hombres y un $3.5 \%$ no se identifican con género femenino ni masculino. 
La segunda pregunta se formuló en relación con el uso de la vacuna contra el COVID-19. Se observa (ver Tabla 4) que las dos respuestas mayoritarias vuelven a ser la A y la D. Sin embargo, aquí el mayor porcentaje de respuestas corresponde con la opción D (154 respuestas que supone el $50 \%$ de las respuestas totales). Si comparamos las respuestas con las respuestas a la pregunta 1 , se observa que ha habido una migración de respuestas hacia la opción de respuesta $D$, disminuyendo el porcentaje de respuestas $A$, en todos los casos salvo de los alumnos de $2^{\circ}$ de ESO que han aumentado las respuestas A en esta segunda pregunta.

Tabla 4. Porcentajes de respuestas a la pregunta 2, en función del curso.

\begin{tabular}{lcccccc}
\hline & $1^{0} \mathrm{ESO}$ & $2^{\mathrm{a} E S O}$ & $3^{\circ} \mathrm{ESO}$ & $4^{\circ} \mathrm{ESO}$ & $1^{\circ}$ Bto & $2^{\circ}$ Bto \\
\cline { 2 - 7 } $\mathrm{A}$ & $42.9 \%$ & $53.6 \%$ & $41.5 \%$ & $35.9 \%$ & $25 \%$ & $33.3 \%$ \\
$\mathrm{~B}$ & $1.2 \%$ & $5.3 \%$ & $7.5 \%$ & $5.1 \%$ & $3.6 \%$ & $4.2 \%$ \\
$\mathrm{C}$ & $1.2 \%$ & $1.8 \%$ & $5.7 \%$ & $0 \%$ & $0 \%$ & $2.1 \%$ \\
$\mathrm{D}$ & $48.8 \%$ & $37.5 \%$ & $45.3 \%$ & $59.0 \%$ & $67.9 \%$ & $54.2 \%$ \\
$\mathrm{E}$ & $5.9 \%$ & $1.8 \%$ & $0 \%$ & $0 \%$ & $3.5 \%$ & $6.2 \%$ \\
\hline
\end{tabular}

Analizando las respuestas atendiendo al género del alumnado, se observa que en la respuesta $A$ la responden más del doble de hombres que de mujeres en caso de los alumnos de 1 y $2^{\circ}$ de ESO y en $1^{\circ}$ Bto. Y esta tendencia se invierte en la respuesta D ("para evitar que te contagies y si te contagias, sanar los síntomas y evitar el contagio a los demás"), que escogen más del doble de mujeres que de hombres en el caso de $1^{\circ}$ de ESO. Del total de respuestas $A$, el $36.8 \%$ son de mujeres, y en el caso de la respuesta $D$, el $40 \%$ de estas respuestas es también de mujeres.

Realizando el análisis global sobre el total de mujeres, el $34.6 \%$ de todas las mujeres elige la opción $A$ y el $60.2 \%$ responde D a esta pregunta 2 , mientras que, sobre el total de hombres, los porcentajes de respuesta $A$ y $D$ son similares (45.7\% elige la opción A, y el $43.2 \%$ la D).

Para analizar la opinión que los alumnos tienen sobre la utilidad actual de las vacunas, se formuló la pregunta 3: ¿Crees que las vacunas son útiles a día de hoy?

De manera contundente, los alumnos creen que las vacunas son útiles ya que evitan la propagación de enfermedades (ver Tabla 5). Analizando las respuestas por curso, en todos los casos la mayoría de los alumnos de cada curso han respondido la opción $A$.

Tabla 5. Porcentajes de respuestas a la pregunta 3, en función del curso.

\begin{tabular}{ccccccc}
\hline & $1^{\circ} \mathrm{ESO}$ & $2^{\mathrm{a} E S O}$ & $3^{\circ} \mathrm{ESO}$ & $4^{\circ} \mathrm{ESO}$ & $1^{\circ}$ Bto & $2^{\circ}$ Bto \\
\cline { 2 - 7 } $\mathrm{A}$ & $94.0 \%$ & $85.7 \%$ & $100 \%$ & $84.6 \%$ & $100 \%$ & $97.9 \%$ \\
$\mathrm{~B}$ & $4.8 \%$ & $7.1 \%$ & $0 \%$ & $12.8 \%$ & $0 \%$ & $0 \%$ \\
$\mathrm{C}$ & $0 \%$ & $1.7 \%$ & $0 \%$ & $0 \%$ & $0 \%$ & $0 \%$ \\
$\mathrm{D}$ & $1.2 \%$ & $5.5 \%$ & $0 \%$ & $2.6 \%$ & $0 \%$ & $2.1 \%$ \\
\hline
\end{tabular}

Si analizamos las respuestas según género, vemos que la respuesta $A$, la han elegido hombres y mujeres, en porcentaje similar $(44.8 \%$ de la respuesta $A$ corresponde a mujeres, el $52.5 \%$ de la respuesta $\mathrm{A}$, la han elegido hombres y el $2.7 \%$ otro género). 
Y la última pregunta de esta categoría, formulada para valorar la percepción de los encuestados ante las vacunas es la siguiente: ¿cuál es tu opinión sobre la vacuna contra el Covid?

Las respuestas a esta pregunta muestran una gran dispersión. Como se puede observar en la tabla 6, las respuestas mayoritarias en todos los cursos, salvo en 4ํㅡㄹ ESO donde la mayoría "no tiene opinión sobre la vacuna", son: "Creo que la vacuna es segura porque ha pasado muchos controles sanitarios" y "Creo que no se conocen los efectos adversos de la vacuna y puede ser peligrosa".

Tabla 6. Porcentajes de respuestas a la pregunta 4, en función del curso.

\begin{tabular}{lcccccc}
\hline & $1^{\circ} \mathrm{ESO}$ & $2^{\mathrm{a} E S O}$ & $3^{\circ} \mathrm{ESO}$ & $4^{\circ} \mathrm{ESO}$ & $1^{\circ}$ Bto & $2^{\circ}$ Bto \\
\hline $\mathrm{A}$ & $19.0 \%$ & $23.2 \%$ & $34.0 \%$ & $20.5 \%$ & $46.4 \%$ & $37.5 \%$ \\
$\mathrm{~B}$ & $36.9 \%$ & $30.4 \%$ & $20.7 \%$ & $17.9 \%$ & $28.6 \%$ & $33.3 \%$ \\
$\mathrm{C}$ & $23.8 \%$ & $14.3 \%$ & $5.7 \%$ & $15.4 \%$ & $7.1 \%$ & $0 \%$ \\
$\mathrm{D}$ & $3.6 \%$ & $14.3 \%$ & $1.9 \%$ & $15.4 \%$ & $0 \%$ & $2.1 \%$ \\
$\mathrm{E}$ & $16.7 \%$ & $17.8 \%$ & $37.7 \%$ & $30.8 \%$ & $17.9 \%$ & $27.1 \%$ \\
\hline
\end{tabular}

Atendiendo a la distribución de respuestas de acuerdo al género, se observa que la mayoría de los alumnos que contestan la opción C, ("Dudo que la vacuna sea eficaz y además puede ser peligrosa") supone un $30 \%$ de hombres de $1^{\circ}$ de ESO.

\section{Categoría 2: Percepción del Riesgo}

Para analizar la percepción ante el riesgo de contagio del virus, se formularon la pregunta 5 (¿crees que tienes riesgo de contagio frente al COVID-19?) y la cuestión 6 (indica qué situación crees que es de riesgo para contagiarse de COVID-19).

Las respuestas muestran que en todos los cursos hay percepción del riesgo de contagio, ya que, en todos, un $25 \%$ o más de los encuestados responden que "sí, creo que tengo riesgo de contagiarme". Pero al mismo tiempo, la otra respuesta mayoritaria en todos los casos es la $\mathrm{C}$, que indica que "no tienen riesgo de contagiarse porque cumplen con las normas de seguridad.

Tabla 7. Porcentajes de respuestas a la pregunta 5 , en función del curso.

\begin{tabular}{lcccccc}
\hline & $1^{0} \mathrm{ESO}$ & $2^{\mathrm{a} E S O}$ & $3^{\circ} \mathrm{ESO}$ & $4^{\circ} \mathrm{ESO}$ & $1^{\circ}$ Bto & $2^{\circ}$ Bto \\
\cline { 2 - 7 } $\mathrm{A}$ & $29.8 \%$ & $25 \%$ & $41.5 \%$ & $38.5 \%$ & $39.3 \%$ & $47.9 \%$ \\
$\mathrm{~B}$ & $5.9 \%$ & $8.9 \%$ & $1.9 \%$ & $10.3 \%$ & $10.7 \%$ & $0 \%$ \\
$\mathrm{C}$ & $59.5 \%$ & $53.6 \%$ & $41.5 \%$ & $33.3 \%$ & $50 \%$ & $31.2 \%$ \\
$\mathrm{D}$ & $3.6 \%$ & $7.1 \%$ & $11.3 \%$ & $12.8 \%$ & $0 \%$ & $6.3 \%$ \\
$\mathrm{E}$ & $1.2 \%$ & $5.4 \%$ & $3.8 \%$ & $5.1 \%$ & $0 \%$ & $14.6 \%$ \\
\hline
\end{tabular}

La distribución de respuestas según el género en esas dos preguntas es equivalente, es decir, la contestan en la misma medida hombres, mujeres y personas identificadas con otros géneros. Aquí es interesante ver que la mayoría de los que eligen la opción $\mathrm{E}$ "No tengo riesgo de contagiarme porque ya he tenido COVID" son varones de $2^{\circ}$ de Bachillerato.

En relación con las situaciones que creen de riesgo para contagiarse del COVID-19 (pregunta 6), tal como se ve en la Tabla 8, todos los grupos de alumnos creen que hay riesgo en cualquiera de las situaciones planteadas, con o sin uso de mascarilla. Pero llama especialmente la atención el caso de los alumnos de $4^{\circ} \mathrm{ESO}$, en el que un $30 \%$ dan la respuesta $E$ "Ninguna de las situaciones es de riesgo", la mayoría del alumnado que responde esta opción es de género femenino. 
Tabla 8. Porcentajes de respuestas a la pregunta 6 , en función del curso.

\begin{tabular}{ccccccc}
\hline & $1^{\circ} \mathrm{ESO}$ & $2^{\mathrm{a}} \mathrm{ESO}$ & $3^{\circ} \mathrm{ESO}$ & $4^{\circ} \mathrm{ESO}$ & $1^{0} \mathrm{~B}$ to & $2^{\circ}$ Bto \\
\cline { 2 - 7 } $\mathrm{A}$ & $31 \%$ & $19.6 \%$ & $24.5 \%$ & $23.1 \%$ & $7.1 \%$ & $8.3 \%$ \\
$\mathrm{~B}$ & $3.6 \%$ & $3.6 \%$ & $1.9 \%$ & $0 \%$ & $3.6 \%$ & $0 \%$ \\
$\mathrm{C}$ & $20.2 \%$ & $39.3 \%$ & $26.4 \%$ & $17.9 \%$ & $50 \%$ & $29.2 \%$ \\
$\mathrm{D}$ & $32.1 \%$ & $33.9 \%$ & $47.2 \%$ & $28.2 \%$ & $39.3 \%$ & $58.3 \%$ \\
$\mathrm{E}$ & $13.1 \%$ & $3.6 \%$ & $0 \%$ & $30.8 \%$ & $0 \%$ & $4.2 \%$ \\
\hline
\end{tabular}

\section{Categoría 3: Actitudes ante la vacuna frente al COVID-19}

Para analizar la actitud ante la vacuna se formularon las preguntas 7 (¿te pondrías la vacuna contra el COVID?), 8 (¿aceptarías vacunarte contra el COVID?), 9 (Sí tu respuesta a la pregunta 8 ha sido sí: ¿Por qué?) y 10 (Sí tu respuesta a la pregunta 8 ha sido no: ¿Por qué?).

A continuación, se muestran las respuestas a las preguntas agrupadas en una misma tabla.

Tabla 9. Porcentajes de respuestas a las preguntas 7, 8, 9 y 10, en función del curso.

\begin{tabular}{|c|c|c|c|c|c|c|}
\hline Pregunta 7 & $1^{\circ} \mathrm{ESO}$ & $2^{\mathrm{a}} \mathrm{ESO}$ & $3^{\circ} \mathrm{ESO}$ & $4^{\circ} \mathrm{ESO}$ & $1^{\circ}$ Bto & $2^{\circ} \mathrm{Bto}$ \\
\hline$A$ & 13.0 & 14.3 & 22.6 & 10.2 & 32.2 & 40.4 \\
\hline$B$ & 41.7 & 37.5 & 22.6 & 12.8 & 42.8 & 17.0 \\
\hline C & 15.5 & 10.7 & 7.6 & 2.6 & 7.1 & 4.3 \\
\hline D & 1.2 & 3.6 & 0 & 23.1 & 0 & 0 \\
\hline$E$ & 28.6 & 33.9 & 47.2 & 51.3 & 17.9 & 38.3 \\
\hline Pregunta 8 & $1^{\circ} \mathrm{ESO}$ & $2^{\mathrm{a}} \mathrm{ESO}$ & $3^{\circ} \mathrm{ESO}$ & $4^{\circ} \mathrm{ESO}$ & $1^{\circ} \mathrm{Bto}$ & $2^{\circ} \mathrm{B}$ to \\
\hline$A$ & 65.5 & 69.3 & 92.5 & 66.7 & 71.4 & 93.7 \\
\hline B & 34.5 & 30.7 & 7.5 & 33.3 & 28.6 & 6.3 \\
\hline Pregunta 9 & $1^{\circ} \mathrm{ESO}$ & $2^{\mathrm{a}} \mathrm{ESO}$ & $3^{\circ} \mathrm{ESO}$ & $4^{\circ} \mathrm{ESO}$ & $1^{\circ} \mathrm{Bto}$ & $2^{\circ} \mathrm{B}$ to \\
\hline A & 11.3 & 16.7 & 8.2 & 14.8 & 16.7 & 20.4 \\
\hline$B$ & 51.6 & 57.1 & 63.3 & 48.2 & 55.6 & 52.3 \\
\hline C & 14.5 & 16.7 & 12.2 & 7.4 & 0 & 2.3 \\
\hline D & 22.6 & 9.5 & 16.3 & 29.6 & 27.7 & 25 \\
\hline Pregunta10 & $1^{\circ} \mathrm{ESO}$ & $2^{\mathrm{a}} \mathrm{ESO}$ & $3^{\circ} \mathrm{ESO}$ & $4^{\circ} \mathrm{ESO}$ & $1^{\circ} \mathrm{Bto}$ & $2^{\circ} \mathrm{B}$ to \\
\hline$A$ & 21.0 & 19.0 & 60.0 & 31.6 & 0 & 33.3 \\
\hline$B$ & 21.1 & 14.3 & 0 & 10.5 & 0 & 16.7 \\
\hline C & 55.3 & 52.4 & 20.0 & 31.6 & 100.0 & 50.0 \\
\hline D & 2.6 & 14.3 & 20.0 & 26.3 & 0 & 0 \\
\hline
\end{tabular}

Las respuestas a la pregunta 7 (¿te pondrías la vacuna contra el COVID?) indican lo siguiente. Mayoritariamente, los alumnos creen que deben esperar un tiempo para ver los efectos en otras personas (respuesta E). Principalmente son los alumnos de $2^{\circ}, 3^{\circ}$ y $4^{\circ}$ de ESO los que esperarían antes de ponerse la vacuna para confirmar en otras personas, posibles efectos secundarios. También en $2^{\circ}$ de Bachillerato un alto porcentaje de alumnos (38.3\%) comparten esa opinión. Y, por otro lado, son los alumnos de cursos de Bachillerato los que en porcentajes más altos eligen la respuesta A (sí, es necesario vacunarme). Son los alumnos de los primeros cursos ( $1^{\circ}$ y $2^{\circ}$ de ESO, aunque también $4^{\circ}$ ) los que eligen la respuesta $B$ (Necesito tener más información para saber si debo vacunarme).

Cuando la pregunta se formula en relación a la vacunación frente al virus (pregunta 8: "¿Aceptarías vacunarte frente al COVID?") las respuestas indican que, en todos los cursos, la mayoría de los alumnos aceptarían vacunarse, siendo en $3^{\circ}$ de ESO y en $2^{\circ}$ de Bachillerato donde la aceptación es mayor, superando el $90 \%$ en ambos cursos. 
Tras analizar las respuestas a la pregunta 7 según el género, vemos que, mayoritariamente son hombres de $2^{\circ}$ de Bachillerato los que escogen la respuesta $A$, mientras que los que eligen esperar para ver antes los efectos en otras personas son hombres y mujeres a partes iguales. Sin embargo, en 4ํ de ESO, los que eligen esa opción, son mayoritariamente mujeres.

Para conocer los motivos que, a los estudiantes, les llevarían a vacunarse o a no vacunarse, se formularon las preguntas 9 y 10, respectivamente. Los alumnos que contestaron que estarían dispuestos a vacunarse, eligieron mayoritariamente en todos los cursos, como razón para hacerlo la respuesta B "Aunque tenga algo de miedo a los efectos adversos, creo que evitará peligros mayores". Siendo la distribución entre géneros en esta respuesta igual entre hombres y mujeres.

Y en el caso de haber respondido que no aceptarían vacunarse, los alumnos de todos los cursos, salvo de $3^{\circ}$ de ESO, responden que no se vacunarían porque creen que la vacuna no es eficaz y que no se han hecho suficientes pruebas. En el caso de los alumnos de $1^{\circ}$ de Bachillerato en el que el $100 \%$ de los casos han elegido esta opción. En $3^{\circ}$ y $4^{\circ}$ de ESO, los porcentajes más altos son para la opción A (No creo que me contagie de COVID). Y es en estos dos cursos donde mayores porcentajes obtiene la opción D (La vacuna es una estafa de los gobiernos y las farmacéuticas). También en esta pregunta, la dispersión del género de los estudiantes que la responden es equitativa.

\section{Conclusiones}

A continuación, se discuten los resultados analizando las tres categorías establecidas inicialmente.

En primer lugar, se observa que los alumnos encuestados, en su mayor parte, conocen el uso de las vacunas en general, sin embargo, creen que la vacuna contra el COVID19 sirve para algo distinto que el resto de las vacunas. Se llega a esta conclusión viendo la variación de resultados entre las preguntas 1 y 2 . Cuando a los alumnos se les pregunta por el uso de la vacuna contra la COVID-19, mayoritariamente perciben que esta vacuna sirve para sanar síntomas y evitar contagios a los demás (además de evitar el contagio), a diferencia de lo que pensaban de las vacunas generales de las que opinan que atenúan los síntomas una vez contagiados (además de evitar el contagio). Sorprenden los datos de los alumnos de $1^{\circ}$ Bto donde se observa una migración de respuestas comparando las respuestas de esas dos preguntas 1 y 2 . Cabría esperar que los alumnos de cursos superiores, más formados en ciencia escolar que los de cursos inferiores, mostraran percepciones más críticas. En relación al análisis según género, son las mujeres (un $60.2 \%$ del total de mujeres) más que los hombres $(43.2 \%$ del total de hombres) quienes optan por la respuesta $\mathrm{D}$, que se refiere a evitar el contagio propio y ajeno y a sanar los síntomas.

Del análisis de las respuestas a las preguntas 3 y 4 se puede concluir que, al hablar de vacunas los alumnos perciben con contundencia la utilidad de las mismas, sin embargo, al preguntarles por la vacuna contra el COVID-19 las opiniones se distribuyen mayoritariamente entre alumnos que creen que es segura porque ha pasado numerosos controles y otros que perciben la peligrosidad de no conocer los efectos adversos de la misma.

Estos resultados iniciales sobre la percepción de los alumnos ante la vacuna del COVID19 parecen corroborar los resultados encontrados por Puig, Blanco-Anaya y PérezMaceira (2021) que concluyeron que las noticias falsas, recibidas durante este tiempo 
de pandemia, parecen condicionar a los alumnos frente a la vacuna del COVID-19, desencadenando en los alumnos desconfianza hacia dicha vacuna (Rodríguez-Losada et al., 2021).

Al analizar la percepción de riesgo que tienen los alumnos (categoría 2), los resultados muestran como los alumnos mayoritariamente muestran dos percepciones que a su vez son completamente opuestas. Principalmente son los alumnos de cursos superiores los que perciben que tienen riesgo de contagiarse y son los alumnos de cursos inferiores los que perciben que, si cumplen las normas establecidas, no tienen riesgo de contagio. Cabe destacar que un $14.6 \%$ de los alumnos de $2^{\circ}$ Bto (mayoritariamente alumnos de género masculino) creen que no tienen riesgo de contagio por haber pasado la enfermedad. Con respecto a las situaciones de riesgo, los alumnos perciben riesgo en todas las situaciones planteadas, sin embargo, un porcentaje elevado (30\%) de los alumnos de $4^{\circ}$ ESO (principalmente, alumnas) no perciben riesgo en ninguna de ellas.

Al analizar la actitud ante la vacuna (categoría 3), el alumnado muestra su interés por vacunarse frente al COVID-19 pero en general optarían por esperar a tener más evidencias sobre los efectos que provoca la vacuna o disponer de más información para elegir vacunarse o no. Sin embargo, ante la perspectiva de vacunarse o no vacunarse, los alumnos optarían (toma de decisión en el caso de los adultos e intención de conducta en el caso de los menores) por vacunarse, y lo justifican poniendo por delante del miedo que les supone, los beneficios generales hipotéticos. A pesar de eso, los alumnos que no se vacunarían declaran que evitarían la vacuna porque no creen que se vayan a contagiar o bien porque no la creen eficaz.

Estos últimos resultados podrían responder a la percepción que tienen los alumnos del proceso de desarrollo y validación de la vacuna. López-Goñi (2020) concluyó que los alumnos creen que se ha utilizado "ciencia express" para conseguirla. Esto unido a las opiniones no fundamentadas expresadas en los medios de comunicación han influido en la percepción y actitud del alumnado frente a la enfermedad y la vacunación.

Por último, retomar el objetivo en esencia de este trabajo que ha sido conocer las actitudes del alumnado de secundaria ante la vacuna y la vacunación, mediante una herramienta validada por expertos en un momento transitorio (y probablemente efímero) en el que no existe un sesgo por conocimiento sobre los efectos de la vacuna. Esto refuerza el conocimiento epistemológico de la percepción del alumnado ante cuestiones sociocientíficas de actualidad y presentes en el debate público en todas sus escalas, también dentro del aula. A su vez, se busca abrir la puerta a futuros estudios, que, en comparativa con los resultados de este trabajo y otros similares, permitan establecer qué factores afectan a las actitudes del alumnado hacia la ciencia y en qué medida, sobre todo, aquellos que permitan arrojar luz sobre la influencia de la enseñanzaaprendizaje en ciencias en el aula frente al aprendizaje por otros medios no contrastados.

\section{Referencias}

Acebedo-Díaz, JA y García-Carmona, A (2017). Controversias en la historia de la ciencia y cultura científica. Madrid: Los libros de la Catarata.

Acebedo-Díaz, JA, Vázquez, A, Martín-Gordillo, M, Oliva, JM, Acebedo, P, Paixao, MF y Manassero, MA (2005). Naturaleza de la ciencia y educación científica para la participación ciudadana. Una revisión crítica. Revista Eureka sobre Enseñanza y Divulgación de las Ciencias, 2(2), 155-166.
Blanco, A, España, E y Rodríguez, F (2012). Contexto y enseñanza de la competencia científica. Alambique: Didáctica de las ciencias experimentales, 70, 9-18.

De Pro, A y Pérez, A (2014). Actitudes de los alumnos de primaria y secundaria ante la visión dicotómica de la Ciencia. Enseñanza de las Ciencias, 32(3), 111-132. 
Díaz, N y Jiménez-Liso, MR (2012). Las controversias sociocientificas: Temáticas e importancia para la educación científica. Revista Eureka sobre Enseñanza y Divulgación de las Ciencias, 9, 54-70.

Duit, R, \& Pfund, H (1998). Biliography "Students' alternative frameworks and science education". Kiel: IPN.

Fontes de Gracia, S, García-Gallego, C, Garriga, AJ, Pérez Llantada, MC y Sarriá, E (2008). Diseños de investigación en psicología. Madrid: UNED.

Gardner, PL (1975). Attitudes to science. Studies in Science Education, 2, 1-41.

George, $R$ (2006). A cross-domain analysis of change in students' attitudes toward science and attitudes about the utility of science. International Journal of Science Education, 28(6), 571-589. https://doi.org/10.1080/09500690500338755

Girón-Gambero, J, Lupión-Cobos, T y Blanco-López, A (2020). Desempeño en competencias de estudiantes de $2^{\circ}$ de ESO en una propuesta $\mathrm{ABP}$ sobre la contaminación acústica. En T SolaMartínez, M García-Carmona, A Fuentes-Cabrera, AM RodríguezGarcía y J López-Belmonte. Innovación educativa en la sociedad digital (106-118). Madrid: Dykinson.

Hodson, D (2020). Going beyond STS Education: building a curriculum for sociopolitical activism. Canadian Journal of Science, Mathematics and Technology Education. https://doi.org/10.1007/s42330-020-00114-6

Jiménez-Aleixandre, MP (2010). 10 ideas clave. Competencia en argumentación y uso de pruebas. Barcelona: Graó.

Klopfer, LE (1971). Evaluation of learning in science. In BS Bloom, JT Hastings, \& GF Madaus (Eds.). Handbook of formative and summative evaluation of student learning. London: McGrw-Hill.

López-Goñi, I (2020). Preparados para la próxima pandemia. Reflexiones desde la ciencia. Barcelona: Destino.

Lupión Cobos, T y Girón Gambero, JR (2020). Percepciones del alumnado de Educación Secundaria (15-17 años) hacia la función social de la ciencia. Didáctica de las Ciencias Experimentales y Sociales, 39, 81-98. https://doi.org/10.7203/DCES.39.17766

Mazas, B y Bravo Torija, B (2018). Actitudes hacia la ciencia del profesorado en formación de educación infantil y educación primaria. Profesorado. Revista de Currículum y Formación de Profesorado, 22(2), 285-304.

Morales, P (2000). Medición de actitudes en Psicología y Educación, construcción de escalas y problemas metodológicos $\left(2^{\mathrm{a}}\right.$ Ed.). Madrid: Universidad Pontificia Comillas.

Osborne, J, Simon, S, \& Collins, S (2003). Attitudes towards science: A review of the literature and its implications. International Journal of Science Education, 25(9), 1049-1079. DOl:https://doi.org/10.1080/0950069032000032199

Puig, B, Blanco-Anaya, P, \& Pérez-Maceira, JJ (2021). Fake news or real science? Critical thinking to assess information on COVID19. Frontiers in Education-STEM Education. https://doi.org/10.3389/feduc.2021.646909
Reiss, MJ (2004). Students' attitudes towards science: A long-term perspective. Canadian Journal of Science Mathematics and Technology Education, 89-101. https://doi.org/10.1080/14926150409556599

Rodríguez-Losada, N, Puig, B, Cebrian-Robles, D y Blanco-López, A (2021). La toma de decisiones responsables frente a la vacuna de la COVID-19. Conocimientos y posiciones de futuros docentes. Revista Internacional de Pesquisa em Didáctica das Cièncias e Matemática, 2, 1-15.

Sadler, TD (2011). Socio-scientific issues in the classroom. Dordrecht: Springer.

Sadler, TD, \& Zeidler, DL (2005). Patterns of informal reasoning in the context of socioscientific decision making. Journal of Research in Science Teaching, 42, 112-128. https://doi.org/10.1002/tea.20042

Solbes, J, Furió, C, Domínguez, MC, Fernández, J, Tarín, F, \& Guisasola, J (2012). What factors have an influence on a quality teaching practice in Sciences? Procedia-Social and Behavioral Sciences, $\quad 46, \quad 4513-4517$. https://doi.org/10.1016/j.sbspro.2012.06.287

Toma, RB (2020). Revisión sistemática de instrumentos de actitudes hacia la ciencia (2004-2016). Enseñanza de las Ciencias, 38(3), 143-159. https://doi.org/10.5565/rev/ensciencias.2854

Uskola, A, Burgoa, B y Maguregi, G (2021). Integración del conocimiento científico y de la capacidad argumentativa en tomas de decisión sobre temas sociocientíficos. Revista Eureka sobre Enseñanza y Divulgación de las Ciencias, 18(1), 1101. https://doi.org/10.25267/Rev_Eureka_ensen divulg_cienc.2021.v1 8.11.1101

Varela, A, López-Gómez, S y Fraguela-Vale, R (2021). Juego y tareas escolares: el papel de la escuela y la familia en tiempos de confinamiento por la COVID-19. Estudios sobre Educación. https://doi.org/10.15581/004.41.001

Vázquez, A, Acevedo, JA y Manassero, MA (2005). Más allá de la enseñanza de las ciencias para científicos: hacia una educación científica humanística. Revista Eureka de Enseñanza y Divulgación de las Ciencias, 4(2), 1-30.

Vázquez, A y Manassero, MA (2008). El declive de las actitudes hacia la ciencia de los estudiantes: un indicador inquietante para la educación científica. Revista Eureka de Enseñanza y Divulgación de las Ciencias, 5(3), 274-292.

Vázquez, A y Manassero, MA (2009). Patrones actitudinales de la vocación científica y tecnológica en chicas y chicos de secundaria. Revista lberoamericana de Educación, 50(4), 1-15. 